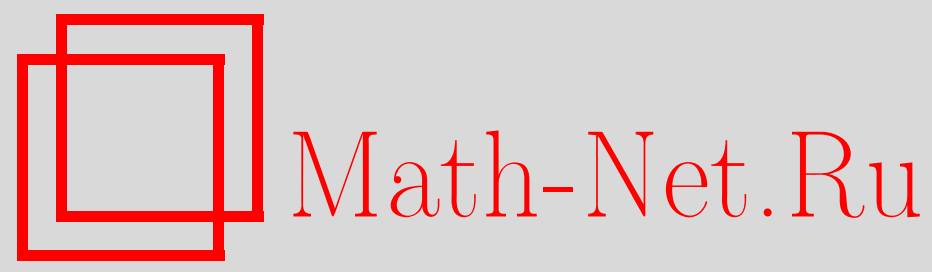

С. В. Конягин, О возвращаемости интеграла нечетной условнопериодической функции, Матем. заметки, 1997, том 61, выпуск 4, 570-577

DOI: https://doi.org/10.4213/mzm1535

Использование Общероссийского математического портала Math-Net.Ru подразумевает, что вы прочитали и согласны с пользовательским соглашением http://www.mathnet.ru/rus/agreement

Параметры загрузки:

IP : 54.205 .225 .156

26 апреля 2023 г., 13:57:11

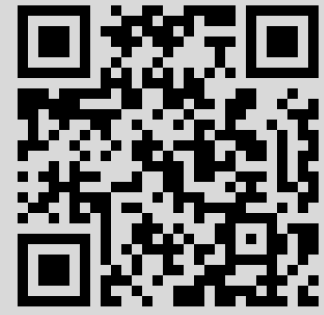




\section{О ВОЗВРАЩАЕМОСТИ ИНТЕГРАЛА НЕЧЕТНОЙ УСЛОВНОПЕРИОДИЧЕСКОЙ ФУНКЦИИ}

\section{С. В. Конягин}

Доказана возвращаемость интеграла достаточно гладкой нечетной условнопериодической функции с нулевым средним и несоизмеримым набором частот. При этом указаны оценки снизу и сверху на гладкость, гарантирующую возвращаемость.

Библиография: 12 названий.

Введение. В настоящей работе рассматривается поведение интеграла

$$
I(t)=\int_{0}^{t} f\left(\omega_{1} s, \ldots, \omega_{d} s\right) d s
$$

где функция $f\left(x_{1}, \ldots, x_{d}\right)$ непрерьвна на торе $\mathbb{T}^{d}$,

$$
\int_{\mathbb{T}^{d}} f\left(x_{1}, \ldots, x_{d}\right) d x=0
$$

а частоты $\omega_{1}, \ldots, \omega_{d} \in \mathbb{R}$ линейно независимы над $\mathbb{Z}$. Этот интеграл ввел в рассмотрение А. Пуанкаре [1] при анализе возмущений в небесной механике. Согласно гипотезе В.В. Козлова при достаточной гладкости функции $f$ интеграл $I(t)$ возвращается, т.е.

$$
\forall \varepsilon>0, \quad \forall T>0 \quad \exists t>T: \quad|I(t)|<\varepsilon .
$$

В случае $d=2$ возврашаемость имеет место для всех несоизмеримых частот $\omega_{1}, \omega_{2}$ при условии абсолютной непрерывности функции $f$ (см. [2], а также [3, гл. 8]). При $d=3$ гипотеза В. В. Козлова доказана Н. Г. Мощевитиным [4], возвращаемость имеет место для $f \in C^{28}\left(\mathbb{T}^{3}\right)$. В то же время, в [1], [3] построен пример непрерьвной функции $f\left(x_{1}, x_{2}\right)$ и набора частот $\omega_{1}=1, \omega_{2}=\sqrt{2}$ таких, что соответствующий интеграл $I(t)$ стремится $\mathrm{K}$ бесконечности при $t \rightarrow \infty$. Н.Г. Мощевитин [5] для произвольного $d \geqslant 2$ построил пример невозвращаемости с $f \in C^{d-2}\left(\mathbb{T}^{d}\right)$. В общем случае для бесконечно дифференцируемых или аналитических $f$ гипотеза В. В. Козлова пока не доказана и не опровергнута. Некоторые результаты в этом направлении имеются в [6]-[12].

В данной работе мы ограничиваемся нечетными функциями $f$. Мы докажем следующие теоремы.

Работа выполнена при финансовой поддержке Российского фонда фундаментальных исследований, грант № 96-01-00378. 
Teорема 1. При $d \geqslant 3, m=3 d \cdot 2^{d-1}, f \in C^{m}\left(\mathbb{T}^{d}\right), f(-x)=-f(x)$, возвращаемость имеет место.

TEOPEMA 2. Прu $d \geqslant 4$,

$$
m=\left[\frac{2^{d-1}(d-2)^{d-2}}{(d-1)^{d-1}}\right]-1
$$

существуют функция $f \in C^{m}\left(\mathbb{T}^{d}\right), f(-x)=-f(x)$, и частоты $\omega_{1}, \ldots, \omega_{d}$ такие, что $I(t) \rightarrow \infty$ при $t \rightarrow \infty$.

(Квадратные скобки обозначают целую часть.)

Заметим, что число $m$ в теореме 2 имеет порядок $2^{d} / d$, причем при $d \geqslant 9$ справедливо неравенство $m>d-2$, т.е. теорема 2 показывает возможность невозвращаемости при большей гладкости на функцию, чем в [5].

Размерность $d$ мы считаем фиксированной, так что положительные константы в по-

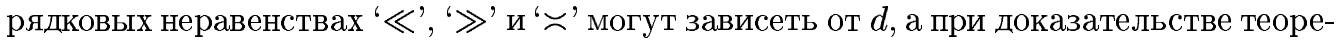
мы 1 , кроме того, от $f$ и $\omega=\left(\omega_{1}, \ldots, \omega_{d}\right) \in \mathbb{R}^{d}$. Через $\|x\|$ будем обозначать расстояние от действительного числа $x$ до ближайшего целого. Всюду ниже $k=\left(k_{1}, \ldots, k_{d}\right) \in \mathbb{Z}^{d}$, $|k|=\max \left(\left|k_{1}\right|, \ldots,\left|k_{d}\right|\right)$ - норма вектора $k$.

1. ДоказАТЕЛЬСТво тЕоремы 1 . Мы считаем, что функция $f$ удовлетворяет условию (1) и требованиям теоремы $1, a \omega_{1}, \ldots, \omega_{d}$ линейно независимы над $\mathbb{Z}$. Рассмотрим разложение $f$ в ряд Фурье:

$$
f(x)=\sum_{k} b_{k} \sin \langle k, x\rangle
$$

При этом мы будем считать, что $b_{k}=0$ при $\langle k, \omega\rangle \leqslant 0$; мы вправе это делать в силу линейной независимости $\omega_{1}, \ldots, \omega_{d}$. Положим $r=m-d / 2>0$.

Лемма 1. При $T \rightarrow \infty$

$$
T \sum_{|k|>T^{1 / r}}\left|b_{k}\right| \rightarrow 0
$$

ДокАЗАТЕЛЬСтво. Из непрерывности $\partial^{m} f / \partial x_{j}^{m}(j=1, \ldots, d)$ следует, что

$$
\sum_{k}\left|k_{j}\right|^{2 m}\left|b_{k}\right|^{2}<\infty
$$

Значит,

$$
\sum_{k}|k|^{2 m}\left|b_{k}\right|^{2}<\infty
$$

т.е.

$$
\sum_{|k|>K}|k|^{2 m}\left|b_{k}\right|^{2} \rightarrow 0 \quad(K \rightarrow \infty) .
$$

Положим $K=T^{1 / r}$. По неравенству Коши-Буняковского имеем

$$
\left(\sum_{|k|>K}\left|b_{k}\right|\right)^{2} \leqslant \sum_{|k|>K}|k|^{2 m}\left|b_{k}\right|^{2} \sum_{|k|>K}|k|^{-2 m} .
$$


Далее,

$$
\sum_{|k|>K}|k|^{-2 m}=\sum_{l>K} \sum_{|k|=l} l^{-2 m} \ll \sum_{l>K} l^{d-1-2 m} \ll K^{d-2 m} .
$$

Отсюда, из (2) и (3) вытекает утверждение леммы.

Фиксируем $\varepsilon>0$ и, считая $T$ достаточно большим числом (т.е. $T>T(\varepsilon, f, \omega)$ ), мы построим $t=t(T, \varepsilon, f, \omega)$ такое, что $|I(t)|<\varepsilon$ и $t \rightarrow \infty(T \rightarrow \infty)$. Положим $\delta=1 /\left(3 \cdot 2^{d}\right)$, $\Delta=T^{d / r}, \Gamma=\Delta^{-1-\delta}$. Определим набор чисел $\Gamma_{d-j}=\Gamma^{3 \cdot 2^{j}-2}(j=0,1, \ldots, d)$. Заметим, что

$$
\Gamma_{0}>T^{-2}
$$

В самом деле, $\Gamma_{0}=T^{-a}$, где

$$
a=\frac{(1+\delta)\left(3 \cdot 2^{d}-2\right) d}{r}=\frac{2(1+\delta)\left(3 \cdot 2^{d}-2\right)}{\left(3 \cdot 2^{d}-1\right)}<2 .
$$

Пусть

$$
\gamma_{1}=\min _{0<|k| \leqslant K}|\langle k, \omega\rangle|,
$$

тогда $\gamma_{1}>0$ в силу линейной независимости $\omega_{1}, \ldots, \omega_{d}$ над $\mathbb{Z}$. Выберем такое $k^{(1)}$, что $0<\left|k^{(1)}\right| \leqslant K$ и $\left\langle k^{(1)}, \omega\right\rangle=\gamma_{1}$. Предположим, что $j \in\{2, \ldots, d\}$ и векторы $k^{(1)}, \ldots, k^{(j-1)}$ уже построены. Среди всех линейно независимых от них векторов $k$ таких, что $|k| \leqslant K$, выберем такой вектор $k^{(j)}$, что величина $\langle k, \omega\rangle$ положительна и принимает наименьшее возможное значение, которое мы обозначим через $\gamma_{j}$. Таким образом, мы определили линейно независимые векторы $k^{(1)}, \ldots, k^{(d)}$ и числа $\gamma_{1}, \ldots, \gamma_{d}$, причем

$$
0<\gamma_{1} \leqslant \cdots \leqslant \gamma_{d} \leqslant \max _{j}\left|\omega_{j}\right| .
$$

Последнее неравенство в (5) следует из того, что при некотором $j$ базисньй вектор $k$, у которого $j$-я координата равна 1 , а остальные - нулю, не является линейной комбинацией векторов $k^{(1)}, \ldots, k^{(d-1)}$ и, значит, $\gamma_{d} \leqslant\left|\omega_{j}\right|$. Положим также $\gamma_{0}=1 / T^{2}$, $\gamma_{d+1}=+\infty$.

Так как все координаты векторов $k^{(1)}, \ldots, k^{(d)}$ по модулю не превосходят $K$, то определитель $\Delta_{0}$ матрицы, составленной из этих векторов, удовлетворяет неравенству $0<\left|\Delta_{0}\right| \ll \Delta$. Следуюшая лемма немедленно вытекает из правила Крамера и определения чисел $\gamma_{j}$.

Лемма 2. Пусть $|k| \leqslant K u|\langle k, \omega\rangle|<\gamma_{l+1}$ при некотором $l \in\{1, \ldots, d\}$. Тогда найдутся зависящие от $k$ челье числа $\Delta_{1}, \ldots, \Delta_{l}$ такие, что

$$
\Delta_{0} k=\sum_{j=1}^{l} \Delta_{j} k^{(j)}, \quad\left|\Delta_{j}\right| \ll \Delta \quad(j=1, \ldots, l) .
$$

Применяя лемму 2 для вектора $(1,0, \ldots, 0)$, мы имеем

$$
\omega_{1}=\sum_{j=1}^{d} \Delta_{j} \gamma_{j}
$$


откуда и из (5) получаем

$$
\gamma_{d} \gg \Delta^{-1}
$$

Выберем теперь максимальное $n \in\{0, \ldots, d\}$ такое, что $\gamma_{n}<\Gamma_{n}$. Такое $n$ сушествует, так как ввиду (4) $\gamma_{0}<\Gamma_{0}$; с другой стороны, (7) показьвает, что $n<d$. По определению $l$ справедливы неравенства

$$
\gamma_{n}<\Gamma_{n}, \quad \gamma_{n+1} \geqslant \Gamma_{n+1}, \quad \ldots, \quad \gamma_{d} \geqslant \Gamma_{d}
$$

Положим $N_{0}=\left[(\Delta \Gamma)^{-1 / 2}\right]$ (напомним, что квадратные скобки обозначают целую часть), тогда

$$
N_{0} \rightarrow \infty \quad(T \rightarrow \infty)
$$

Заметим, что в силу (5) все числа $\sqrt{\gamma}_{j} \Gamma(j=1, \ldots, d)$ меньше 1 . По принципу Дирихле найдется такое натуральное $N$, что

$$
\left\|\frac{N_{0} N \gamma_{j}}{\gamma_{d}}\right\| \leqslant \sqrt{\gamma}_{j} \Gamma \quad(j=n+1, \ldots, d-1)
$$

и

$$
N \ll \prod_{j=n+1}^{d-1}\left(\sqrt{\gamma}_{j} \Gamma\right)^{-1}
$$

Обозначим $t_{0}=N_{0} N / \gamma_{d}$, тогда (10) перепишется в виде

$$
\left\|t_{0}\left\langle k^{(j)}, \omega\right\rangle\right\| \leqslant \sqrt{\gamma}_{j} \Gamma \quad(j=n+1, \ldots, d),
$$

а из (11) и (8) следует, что

$$
t_{0} \ll N_{0}\left(\Gamma_{d}\right)^{-1} \prod_{j=n+1}^{d-1}\left(\sqrt{\Gamma}_{j} \Gamma\right)^{-1}=N_{0} \Gamma \Gamma_{n}^{-1 / 2} .
$$

Положим $t=2 \pi\left|\Delta_{0}\right| t_{0}$, тогда в силу (5) и (9) $t \rightarrow \infty$ при $T \rightarrow \infty$, и нам осталось проверить, что $|I(t)|<\varepsilon$.

Мы имеем

$$
|I(t)| \leqslant \sum_{k}\left|b_{k}\right| \frac{1-\cos (t\langle k, \omega\rangle)}{\langle k, \omega\rangle}
$$

(напомним, что $\left|b_{k}\right| \neq 0$ только в случае $\langle k, \omega\rangle>0$ ). Сумму в $(14)$ мы будем оценивать отдельно для $k \in(I), k \in(I I)$ и $k \in(I I I)$, где $(I)=\left\{k:|k| \leqslant K, 0<\langle k, \omega\rangle<\gamma_{n+1}\right\}$, $(I I)=\left\{k:|k| \leqslant K,\langle k, \omega\rangle \geqslant \gamma_{n+1}\right\},(I I I)=\{k:|k|>K\}$.

Пусть $k \in(I)$, тогда при некотором $l \in\{1, \ldots, n\}$ справедливо неравенство

$$
\gamma_{l} \leqslant\langle k, \omega\rangle<\gamma_{l+1}
$$

Представив $\Delta_{0} k$ в виде (6), мы получим

$$
\left|\frac{t\langle k, \omega\rangle}{2 \pi}\right|=\left|t_{0} \sum_{j=1}^{l} \Delta_{j}\left\langle k^{(j)}, \omega\right\rangle\right| \ll \Delta t_{0} \sum_{j=1}^{l} \gamma_{j} \ll \Delta t_{0} \gamma_{l},
$$


откуда в силу (5) и (8)

$$
\frac{1-\cos (t\langle k, \omega\rangle)}{\langle k, \omega\rangle} \ll \frac{\left(\Delta t_{0} \gamma_{l}\right)^{2}}{\gamma_{l}}<\left(\Delta t_{0}\right)^{2} \Gamma_{n} .
$$

Подставляя в последнее неравенство оценку (13), получаем

$$
\frac{1-\cos (t\langle k, \omega\rangle)}{\langle k, \omega\rangle} \ll\left(N_{0} \Gamma \Delta\right)^{2} \leqslant N_{0}^{-2} .
$$

Пусть теперь $k \in(I I)$. Вновь выберем такое $l$, что $\gamma_{l} \leqslant\langle k, \omega\rangle<\gamma_{l+1}$, при этом $l \in\{n+1, \ldots, d\}$. Снова пользуясь представлением (6), имеем

$$
\left\|\frac{t\langle k, \omega\rangle}{2 \pi}\right\|=\left\|t_{0} \sum_{j=1}^{l} \Delta_{j}\left\langle k^{(j)}, \omega\right\rangle\right\| \leqslant \sum_{j=1}^{l} \Delta_{j}\left\|t_{0}\left\langle k^{(j)}, \omega\right\rangle\right\|
$$

и, далее, в силу (6), (12) и (5)

$$
\begin{gathered}
\left\|\frac{t\langle k, \omega\rangle}{2 \pi}\right\| \ll \Delta \sum_{j=1}^{n} t_{0} \gamma_{j}+\Delta \sum_{j=n+1}^{l} \sqrt{\gamma}_{j} \Gamma \ll \Delta t_{0} \gamma_{n}+\Delta \sqrt{\gamma}_{l} \Gamma, \\
1-\cos (t\langle k, \omega\rangle) \ll\left(\Delta t_{0} \gamma_{n}\right)^{2}+\Delta^{2} \gamma_{l} \Gamma^{2} .
\end{gathered}
$$

Значит,

$$
\frac{1-\cos (t\langle k, \omega\rangle)}{\langle k, \omega\rangle} \ll \frac{\left(\Delta t_{0} \gamma_{n}\right)^{2}}{\gamma_{l}}+\Delta^{2} \Gamma^{2}<\left(\Delta t_{0}\right)^{2} \Gamma_{n}+\Delta^{2} \Gamma^{2} .
$$

Первое слагаемое в правой части (16), совпадающее с правой частью (15), было оценено при разборе первого случая, а второе не превосходит $N_{0}^{-4}$. Следовательно,

$$
\frac{1-\cos (t\langle k, \omega\rangle)}{\langle k, \omega\rangle} \ll N_{0}^{-2}
$$

С учетом (9) мы вьводим, что при достаточно большом $T$ суммарньй вклад $k \in(I)$ и $k \in(I I)$ не превосходит

$$
\sum_{k \in(I) \cup(I I)}\left|b_{k}\right| \frac{1-\cos (t\langle k, \omega\rangle)}{\langle k, \omega\rangle} \ll \sum_{k}\left|b_{k}\right| N_{0}^{-2}<\frac{\varepsilon}{2} .
$$

При рассмотрении $k \in(I I I)$ каждое слагаемое в (14) оценим тривиально

$$
\frac{1-\cos (t\langle k, \omega\rangle)}{\langle k, \omega\rangle}=\int_{0}^{t} \sin (s\langle k, \omega\rangle) d s \leqslant T .
$$

Заметим, что в силу (13) и (4)

$$
t \ll N_{0} \Delta \Gamma \Gamma_{n}^{-1 / 2} \leqslant N_{0} \Delta \Gamma \Gamma_{0}^{-1 / 2} \leqslant N_{0} \Delta \Gamma T,
$$

поэтому при достаточно большом $T$ справедливо неравенство $t \leqslant T$ и по лемме 1

$$
\sum_{k \in(I I I)}\left|b_{k}\right|<\frac{\varepsilon}{2 T}
$$

Отсюда и из (18) вытекает, что

$$
\sum_{k \in(I I I)}\left|b_{k}\right| \frac{1-\cos (t\langle k, \omega\rangle)}{\langle k, \omega\rangle}<\frac{\varepsilon}{2}
$$

Складьвая полученное неравенство с (17), мы получаем (14). Теорема 1 доказана. 
2. ДоКАЗАТЕЛЬСТво ТЕОРЕМЫ 2. СНачала определим частоты $\omega_{1}, \ldots, \omega_{d}$ и проверим, что они линейно независимы над $\mathbb{Z}$. Положим

$$
\lambda=\frac{2 d-4}{d-1}>1, \quad \omega_{d}=1, \quad \omega_{j}=\sum_{l=0}^{\infty} 2^{-\left[\lambda^{(d-1) l+j}\right]} \quad(j=1, \ldots, d-1) .
$$

Допустим, что частоты линейно зависимы над $\mathbb{Z}$, тогда некоторая нетривиальная целочисленная комбинация чисел $\omega_{1}, \ldots, \omega_{d-1}$ равна целому числу $a$, и мы имеем представление

$$
a=\sum_{l=1}^{\infty} N_{l} 2^{-\left[\lambda^{l}\right]}
$$

причем целочисленная последовательность $\left\{N_{l}\right\}$ равномерно ограничена и содержит бесконечно много ненулевых членов. Выберем такие натуральные числа $M$ и $n_{0}$, что $\max _{l}\left|N_{l}\right| \leqslant 2^{M}-2$ и $\left[\lambda^{l+1}\right]-\left[\lambda^{l}\right] \geqslant M$ при $l \geqslant n_{0}$. Пусть $n \geqslant n_{0}$, тогда число

$$
b_{n}=2^{\left[\lambda^{n}\right]} a-\sum_{l=1}^{n} N_{l} 2^{\left[\lambda^{n}\right]-\left[\lambda^{l}\right]}
$$

является целым. С другой стороны,

$$
\left|b_{n}\right| \leqslant \sum_{l=n+1}^{\infty}\left|N_{l}\right| 2^{\left[\lambda^{n}\right]-\left[\lambda^{l}\right]} \leqslant \sum_{l=n+1}^{\infty}\left(2^{M}-2\right) 2^{-(l-n) M}<1,
$$

т.е. $b_{n}=0$; стало быть, при $n \geqslant n_{0}$

$$
N_{n+1}=2^{\left[\lambda^{n+1}\right]-\left[\lambda^{n}\right]} b_{n}-b_{n+1}=0,
$$

что невозможно.

Так как число

$$
\mu=\frac{2^{d-1}(d-2)^{d-2}}{(d-1)^{d-1}}-1
$$

не целое, то можно найти такое $\varepsilon$, что

$$
0<\varepsilon<\mu-m
$$

Обозначим

$$
\begin{gathered}
A_{j, n}=2^{\left[\lambda^{(d-1) n+j}\right]}, \quad B_{j, n}=\sum_{l=0}^{n} 2^{\left[\lambda^{(d-1) n+j}\right]-\left[\lambda^{(d-1) l+j}\right]} \\
(j=1, \ldots, d-1, \quad n=0,1, \ldots)
\end{gathered}
$$

тогда $A_{j, n} \asymp B_{j, n}$. Теперь мы готовы определить функцию $f$ :

$$
f(x)=\sum_{j=1}^{d-1} \sum_{n=1}^{\infty} A_{j, n}^{-m-\varepsilon} \sin \left(A_{j, n} x_{j}-B_{j, n} x_{d}\right) .
$$


Взяв любую производную порядка не вьше $m$ от ряда Фурье функции $f$, мы получим ряд вида

$$
\sum_{j=1}^{d-1} \sum_{n=1}^{\infty} c_{j, n} \sin \left(A_{j, n} x_{j}-B_{j, n} x_{d}+\theta_{j, n}\right)
$$

где $\left|c_{j, n}\right| \leqslant A_{j, n}^{-m-\varepsilon}\left(A_{j, n}+B_{j, n}\right)^{m} \ll A_{j, n}^{-\varepsilon}$. Из последней оценки следует, что ряд (20) абсолютно сходится, и, значит, $f \in C^{m}\left(\mathbb{T}^{d}\right)$.

$$
\begin{aligned}
& \text { Для } j=1, \ldots, d-1, n=0,1, \ldots \text { обозначим } \\
& \qquad \gamma_{j, n}=A_{j, n} \omega_{j}-B_{j, n} \omega_{d}=\sum_{l=n+1}^{\infty} 2^{\left[\lambda^{(d-1) n+j}\right]-\left[\lambda^{(d-1) l+j}\right]} .
\end{aligned}
$$

Для $n \geqslant 1$ мы полагаем также $\gamma_{0, n}=\gamma_{d-1, n-1}$. Справедливы соотношения

$$
\begin{gathered}
\gamma_{j, n}>0, \quad \gamma_{j, n} \asymp A_{j, n}^{1-\lambda^{d-1}}, \\
\gamma_{j-1, n} \asymp A_{j, n}^{1-\lambda^{d-2}} .
\end{gathered}
$$

Представим $I(t)$ в виде суммы

$$
I(t)=\sum_{j=1}^{d-1} \sum_{n=1}^{\infty} A_{j, n}^{-m-\varepsilon} \frac{1-\cos \left(t \gamma_{j, n}\right)}{\gamma_{j, n}} .
$$

Поскольку все слагаемые в (23) неотрицательны, то для доказательства теоремы нам достаточно "выловить" в этой сумме одно большое слагаемое. Пусть $t>\gamma_{1,0}^{-1}$, тогда найдутся такие $j \in\{1, \ldots, d-1\}$ и $n \in\{0,1, \ldots\}$, что $\gamma_{j-1, n}^{-1}<t \leqslant \gamma_{j, n}^{-1}$. Мы имеем

$$
A_{j, n}^{-m-\varepsilon} \frac{1-\cos \left(t \gamma_{j, n}\right)}{\gamma_{j, n}} \asymp A_{j, n}^{-m-\varepsilon} \frac{\left(t \gamma_{j, n}\right)^{2}}{\gamma_{j, n}} \geqslant A_{j, n}^{-m-\varepsilon} \gamma_{j-1, n}^{-2} \gamma_{j, n}
$$

Подставляя оценки (21) и (22), получаем

$$
A_{j, n}^{-m-\varepsilon} \frac{1-\cos \left(t \gamma_{j, n}\right)}{\gamma_{j, n}} \gg A_{j, n}^{1+2 \lambda^{d-2}-\lambda^{d-1}-m-\varepsilon}=A_{j, n}^{\mu-m-\varepsilon} .
$$

В силу (19) правая часть (24) стремится к бесконечности при $t \rightarrow \infty$, поэтому и $I(t) \rightarrow \infty$ $(t \rightarrow \infty)$. Теорема доказана.

Автор благодарит В. В. Козлова и Н. Г. Мощевитина за внимание к работе. 


\section{СПИСОК ЦИТИРОВАННОЙ ЛИТЕРАТУРЫ}

[1] Пуанкаре А. О кривых, определяемых дифференциальньми уравнениями. М.-Л.: Гостехиздат, 1947.

[2] Сидоров Е. А. Об условиях равномерной устойчивости по Пуассону цилиндрических систем // УМН. 1979. Т. 34. №6. С. 184-188.

[3] Козлов В. В. Методы качественного анализа в динамике твердого тела. М.: Изд-во МГУ, 1980.

[4] Мощевитин Н.Г. О возвращаемости интеграла гладкой трехчастотной условнопериодической функции // Матем. заметки. 1995. Т. 58. № 5. С. 723-735.

[5] Мощевитин Н. Г. О невозвращаемости интеграла условнопериодической функции // Матем. заметки. 1991. Т. 49. №6. С. 138-140.

[6] Спринджук В.Г. Асимптотическое поведение интегралов от квазипериодических функций // Дифференц. уравнения. 1967. Т. 3. №6. С. 862-868.

[7] Спринджук В. Г. Квазипериодические функции с неограниченньм неопределенным интегралом // Докл. АН БССР. 1968. Т. 12. №1. С. 5-8.

[8] Peck L. G. On uniform distribution of algebraic numbers // Proc. Amer. Math. Soc. 1953. V. 4. № 1. P. 440-443.

[9] Мощевитин Н. Г. Финальные свойства интегралов от условнопериодических функций, связанных с проблемой малых знаменателей // Вестн. МГУ. Сер. 1. Матем., мех. 1988. № 5. C. $94-96$.

[10] Мощевитин Н. Г. О равномерном распределении дробных долей системы линейных функций // Вестн. МГУ. Сер. 1. Матем., мех. 1990. № 4. С. 26-31.

[11] Мощевитин Н. Г. К вопросу о поведении интеграла условнопериодической функции // Матем. заметки. 1991. Т. 50. № 3. С. 97-106.

[12] Мощевитин Н. Г. Распределение значений линейных функций и асимптотическое поведение некоторых динамических систем // Матем. заметки. 1995. Т. 58. № 3. С. 394-410. 